\title{
L-Fuzzy Sets for Group Linguistic Preference Modeling: An application to assess a firm's performance
}

\author{
Núria Agell, Mónica Sánchez, Francesc Prats
}

\begin{abstract}
Given a finite totally ordered set of linguistic descriptions, the extended set of qualitative labels with different levels of precision $L$ is constructed. In this framework, qualitative descriptions of a given set are $L$-fuzzy sets. A distance between $L$-fuzzy sets is introduced based on the properties of the lattice $L$. An illustrative example in the retail sector applied to assess a firm's overall performance using perceptions of managers in the firm's different departments is presented.
\end{abstract}

Index Terms-Knowledge Management, Decision support, Uncertainty and fuzzy reasoning.

\section{INTRODUCTION}

Several approaches in the fuzzy set framework have been developed to model linguistic preferences [7], [6], [19]. These approaches allow the imprecise and uncertain knowledge that characterizes human preference reasoning to be handled. Some of these approaches involve different levels of precision or multi-granularity in the fuzzy linguistic modeling and are therefore based on a non-totally ordered set of linguistic labels [3], [13], [15]. Linguistic preference modeling has been used in consensus approaches in decision making, an overview of soft consensus models in fuzzy environments can be found in [8].

$L$-fuzzy sets were defined by Goguen [5] as a generalization of the classic fuzzy sets by considering membership functions with range values in a lattice $L$. In this way, the classic fuzzy sets are a special case of the $L$-fuzzy sets when $L=[0,1]$. Topological and metric properties of $L$-fuzzy sets have been analyzed in [9], [18]. In [11], [12] representation theorems for $L$-fuzzy sets can be found. Using $L$-fuzzy set representations, ordered structures have been characterized in [17]. In addition, several studies have addressed the relation between $L$-fuzzy sets and other extensions of fuzzy sets, such as intuitionistic fuzzy sets and interval-valued fuzzy sets [2], [22].

On the other hand, qualitative reasoning was introduced in the 80's [4] to model real-world problems in which only incomplete qualitative knowledge is available [20]. Qualitative order-of-magnitude models are basic theoretical tools for qualitative reasoning involving different levels of precision or multi-granularity [21]. The adaptation of $L$-fuzzy sets theory

Núria Agell is with the Department of Information Systems Management and GREC Group, in Esade - Universitat Ramon Llull, Barcelona, Spain (email: nuria.agell@esade.edu).

Mónica Sánchez and Francesc Prats are with the Department of Applied Mathematics 2 and GREC Group, in Technical University of Catalonia, UPC-BarcelonaTech, Barcelona, Spain (email: \{monica.sanchez;francesc.prats\}@upc.edu).

This work is partially supported by the SENSORIAL Research Project (TIN2010-20966-C02-01,02), funded by the Spanish Ministry of Science and Information Technology. to represent linguistic preferences via qualitative order-ofmagnitude models was introduced in [14].

In this paper, we present a generalization of the existing qualitative order-of-magnitude models, which allows us to define qualitative descriptions of a set in terms of $L$-fuzzy sets, and formalize the concept of distance between qualitative descriptions by means of a multi-granular set of orderof-magnitude labels.

A formal mathematical model is developed to support experts in group decision-making under uncertainty. The proposed model focuses the situation where the group of decision makers has some subgroups, each subgroup composed by members with similar profiles. The perspectives of the different subgroups are analyzed and modeled via an aggregation of linguistic preferences. Distances between qualitative descriptions are used to measure differences between the subgroups. This contributes to measure the risk and assure the validity of the actions derived from a decision outcome.

The rest of this paper is organized as follows: First Section II introduces the theoretical framework for the new approach. Section III presents the new approach for group preference modeling based on an aggregation of qualitative descriptions and the distances among them. An illustrative example in the retail sector is presented in Section IV. Finally, Section $\mathrm{V}$ contains the main conclusions and lines of future research.

\section{Preliminaries: Qualitative Descriptions as $L$-FUZZY SETS}

In this section, we briefly review some basic concepts that will be used in the next sections [1], [14], [16].

\section{A. The lattice $\left(\mathbb{S}_{n}, \sqcup, \cap\right)$}

From here on, let $\mathcal{S}=\left\{a_{1}, \ldots, a_{n}\right\}$ be a finite totally ordered set, with $a_{1}<\ldots<a_{n}$. The following definitions were introduced in [14]:

Definition 1: The basic qualitative labels (or basic labels) over $\mathcal{S}$ are the singletons $\left\{a_{i}\right\}$, with $i \in\{1, \ldots, n\}$.

Definition 2: The qualitative labels (or labels) over $\mathcal{S}$ are the intervals $\left[a_{i}, a_{j}\right]=\left\{x \in \mathcal{S} \mid a_{i} \leq x \leq a_{j}\right\}$, for all $i, j \in$ $\{1, \ldots, n\}$ with $i \leq j$.

Note that the basic labels are labels and the entire set $\mathcal{S}=$ $\left[a_{1}, a_{n}\right]$ is a label. The label $\mathcal{S}$, which is the union of all basic labels, is frequently denoted by the symbol ? and referred to as the "unknown" label: ? $=\mathcal{S}$.

Definition 3: Let $\mathcal{P}(\mathcal{S})$ be the power set of $\mathcal{S}$. The set $\mathbb{S}_{n}^{*} \subseteq \mathcal{P}(\mathcal{S})$ of all of the qualitative labels over $\mathcal{S}$ is called 
the order-of-magnitude qualitative space with granularity $n$ over $\mathcal{S}$ :

$$
\mathbb{S}_{n}^{*}=\left\{\left[a_{i}, a_{j}\right] \mid i, j \in\{1, \ldots, n\}, i \leq j\right\} .
$$

The set $\mathbb{S}_{n}^{*}$ is extended to include the empty set $\emptyset$ to obtain a lattice structure.

Definition 4: The extended set $\mathbb{S}_{n} \subseteq \mathcal{P}(\mathcal{S})$ of qualitative labels over $\mathcal{S}$ is:

$$
\mathbb{S}_{n}=\mathbb{S}_{n}^{*} \cup\{\emptyset\} .
$$

The order relation to be more precise or equal than between qualitative labels, induced by the inclusion $\subseteq$, is defined as follows:

Definition 5: For any qualitative labels $\left[a_{i}, a_{j}\right]$ and $\left[a_{i^{\prime}}, a_{j^{\prime}}\right]$, we say that $\left[a_{i}, a_{j}\right]$ is more precise or equal than $\left[a_{i^{\prime}}, a_{j^{\prime}}\right]$ iff $\left[a_{i}, a_{j}\right] \subseteq\left[a_{i^{\prime}}, a_{j^{\prime}}\right]$, i.e., $i^{\prime} \leq i$ and $j \leq j^{\prime}$.

Example 1: Let us consider a simple example to illustrate the above definitions. Suppose that a firm's performance regarding its market positioning is qualitatively described by means of the set of qualitative labels over $\mathcal{S}$, with

$$
\mathcal{S}=\left\{a_{1}, a_{2}, a_{3}, a_{4}\right\}
$$

where associate linguistic labels are: not very good, moderately good, very good, extremely good.

Then an example of a basic label is $\left\{a_{2}\right\}=$ moderately good and two examples of non-basic labels are $\left[a_{1}, a_{3}\right]=$ not extremely good and $?=\left[a_{1}, a_{4}\right]=$ unknown. The relation to be more precise or equal than among these three labels gives: $\left\{a_{2}\right\} \subseteq\left[a_{1}, a_{3}\right] \subseteq$ ?.

The binary operations on the extended set $\mathbb{S}_{n}$ of qualitative labels: the connected union, $\sqcup$ and the intersection, $\cap$ provide a lattice structure to $\mathbb{S}_{n}$ [14] (the connected union of two qualitative labels is the least element of $\mathbb{S}_{n}$, based on the subset inclusion relation $\subseteq$, that contains both qualitative labels). In Figure 1 the diagram of this lattice is depicted.

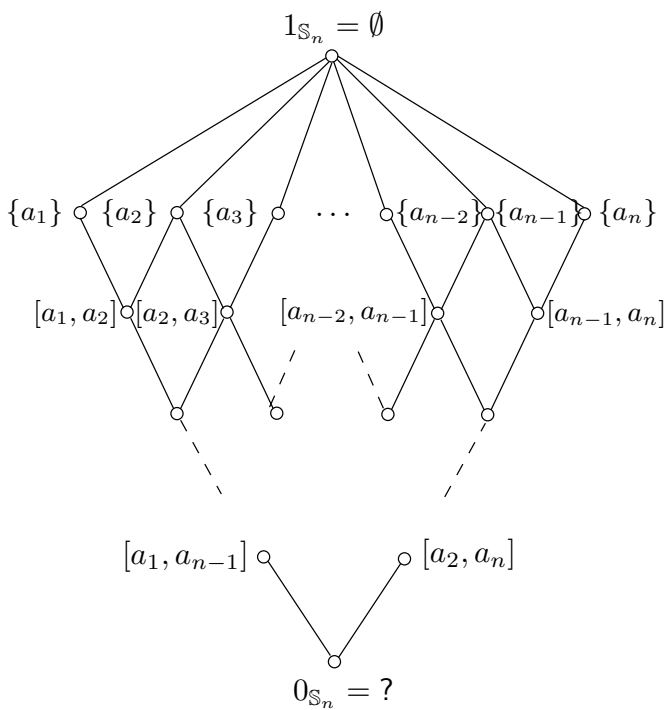

Fig. 1. Diagram of the lattice $\left(\mathbb{S}_{n}, \sqcup, \cap\right)[14]$
A simple calculation proves that the cardinality of $\mathbb{S}_{n}$ is

$$
\left|\mathbb{S}_{n}\right|=1+\frac{n(n+1)}{2} .
$$

The lattice $\left(\mathbb{S}_{n}, \sqcup, \cap\right)$ is not distributive. A counterexample in which the property $X \cap(Y \sqcup Z)=(X \cap Y) \sqcup(X \cap Z)$ does not hold is given in the case where $\mathcal{S}$ has at least three elements, considering $a_{1}, a_{2}, a_{3} \in \mathcal{S}$ such that $a_{1}<a_{2}<a_{3}$ and $Y=\left\{a_{1}\right\}, X=\left\{a_{2}\right\}, Z=\left\{a_{3}\right\}$. In addition, $\left(\mathbb{S}_{n}, \sqcup, \cap\right)$ does not satisfy the modular condition if $n \geq 3$. A sublattice of $\mathbb{S}_{n}$ that is isomorphic to the pentagon lattice is given by the following five labels: $\left[a_{1}, a_{3}\right],\left[a_{1}, a_{2}\right],\left\{a_{1}\right\}, 1_{\mathbb{S}_{n}},\left\{a_{3}\right\}$.

\section{B. Qualitative descriptions as L-fuzzy sets}

The concept of an L-fuzzy set on a non-empty set $\Lambda$ was introduced by Goguen in [5] as a function $f: \Lambda \rightarrow L$, where $L$ is a lattice. This concept is applied to the case of the lattice $\left(\mathbb{S}_{n}, \sqcup, \cap\right)$ of qualitative labels over a finite set $\mathcal{S}$ in the following definitions and theorem.

Definition 6: An $\mathbb{S}_{n}$-fuzzy set on $\Lambda$ is a function $Q: \Lambda \rightarrow$ $\mathbb{S}_{n}$.

Note that any $f: \Lambda \rightarrow\{0,1\}$ defines an ordinary set on $\Lambda$, that is, a subset of $\Lambda$, whose characteristic function is $f$. If $f: \Lambda \rightarrow[0,1]$, then $f$ defines a fuzzy set on $\Lambda$, where for each $\lambda \in \Lambda, f(\lambda)$ is the degree of membership of $\lambda$. We can therefore consider an $\mathbb{S}_{n}$-fuzzy set $Q: \Lambda \rightarrow \mathbb{S}_{n}$ on $\Lambda$ as a set whose elements are assigned qualitative labels from the extended set $\mathbb{S}_{n}$ over $\mathcal{S}$ rather than degrees of membership.

Definition 7: The set $\mathcal{Q}$ of $\mathbb{S}_{n}$-fuzzy sets on $\Lambda$ is:

$$
\mathcal{Q}=\mathbb{S}_{n}{ }^{\Lambda}=\left\{Q \mid Q: \Lambda \rightarrow \mathbb{S}_{n}\right\}
$$

Definition 8: A qualitative description of the set $\Lambda$ by $\mathbb{S}_{n}$ (or using the labels of $\mathbb{S}_{n}$ ) is an $\mathbb{S}_{n}$-fuzzy set on $\Lambda$ such that for all $\lambda \in \Lambda, Q(\lambda)$ is a qualitative label, i.e., $Q(\lambda) \in \mathbb{S}_{n}^{*}=$ $\mathbb{S}_{n}-\{\emptyset\}$.

\section{A distance between qualitative descriptions}

As proved in [14], formula:

$$
D_{\mathbb{S}_{n}^{*}}\left(E_{1}, E_{2}\right)=\operatorname{card}\left(E_{1} \sqcup E_{2}\right)-\operatorname{card}\left(E_{1} \cap E_{2}\right)
$$

for $E_{1}=\left[a_{i}, a_{j}\right]$ and $E_{2}=\left[a_{i^{\prime}}, a_{j^{\prime}}\right]$ in $\mathbb{S}_{n}^{*}$, provides a distance on $\mathbb{S}_{n}^{*}$. This distance between qualitative labels induces a distance between qualitative descriptions.

Let us consider a finite set $\mathrm{Q}=\left\{Q_{1}, \ldots, Q_{k}\right\} \subset \mathbb{S}_{n}^{\Lambda}$ of qualitative descriptions of a set $\Lambda$ by $\mathbb{S}_{n}$. For every $Q_{i} \in \mathrm{Q}$, let $Q_{i}(\Lambda)=\left\{E_{1}^{i}, \ldots, E_{r_{i}}^{i}\right\} \subseteq \mathbb{S}_{n}^{*}$.

Let $\mathcal{P}=\left\{B_{1}, \ldots, B_{m}\right\}$ be the partition of $\Lambda$ such that all functions $Q_{1}, \ldots, Q_{k}$ are constant on each part $B_{t} \in \mathcal{P}, t=$ $1, \ldots, m$ constructed in [14].

Let $Q_{i}\left(B_{t}\right)=\left\{F_{t}^{i}\right\}$, with $F_{t}^{i} \in\left\{E_{1}^{i}, \ldots, E_{r_{i}}^{i}\right\}$, for each $i=1, \ldots, k$ and $t=1, \ldots, m$. Then Formula 1:

$$
D_{Q}\left(Q_{i}, Q_{j}\right)=\sum_{t=1}^{m} \operatorname{card}\left(F_{t}^{i} \sqcup F_{t}^{j}\right)-\sum_{t=1}^{m} \operatorname{card}\left(F_{t}^{i} \cap F_{t}^{j}\right)
$$

provides a distance in Q, i.e., a distance between qualitative descriptions (see [14]). 
Example 2: Let us consider a simple example to illustrate the computation of this distance between qualitative descriptions. Suppose $\Lambda=\left\{\lambda_{1}, \lambda_{2}, \lambda_{3}, \lambda_{4}, \lambda_{5}\right\}$ and $\mathrm{Q}=\left\{Q_{1}, Q_{2}\right\}$, where $Q_{1}$ and $Q_{2}$ are the qualitative descriptions of the set $\Lambda$ by $\mathbb{S}_{4}$ given in Table I.

TABLE I

QUALITATIVE DESCRIPTIONS $Q_{1}$ AND $Q_{2}$

\begin{tabular}{|c|c|c|}
\hline & $Q_{1}$ & $Q_{2}$ \\
\hline$\lambda_{1}$ & {$\left[a_{2}, a_{3}\right]$} & {$\left[a_{3}, a_{4}\right]$} \\
\hline$\lambda_{2}$ & $\left\{a_{4}\right\}$ & $\left\{a_{4}\right\}$ \\
\hline$\lambda_{3}$ & {$\left[a_{2}, a_{3}\right]$} & {$\left[a_{3}, a_{4}\right]$} \\
\hline$\lambda_{4}$ & $\left\{a_{3}\right\}$ & {$\left[a_{3}, a_{4}\right]$} \\
\hline$\lambda_{5}$ & $\left\{a_{4}\right\}$ & {$\left[a_{2}, a_{4}\right]$} \\
\hline
\end{tabular}

Then, the partition of $\Lambda$ such that $Q_{1}$ and $Q_{2}$ are constant on each part is $\mathcal{P}=\left\{B_{1}, \ldots, B_{4}\right\}$, where $B_{1}=$ $\left\{\lambda_{1}, \lambda_{3}\right\}, B_{2}=\left\{\lambda_{2}\right\}, B_{3}=\left\{\lambda_{4}\right\}$ and $B_{4}=\left\{\lambda_{5}\right\}$. Their respective values are:

$F_{1}^{1}=\left[a_{2}, a_{3}\right], F_{2}^{1}=\left\{a_{4}\right\}, F_{3}^{1}=\left\{a_{3}\right\}, F_{4}^{1}=\left\{a_{4}\right\}$,

$F_{1}^{2}=\left[a_{3}, a_{4}\right], F_{2}^{2}=\left\{a_{4}\right\}, F_{3}^{2}=\left[a_{3}, a_{4}\right], F_{4}^{2}=\left[a_{2}, a_{4}\right]$.

Therefore:

$$
\begin{gathered}
D_{\mathrm{Q}}\left(Q_{1}, Q_{2}\right)=\sum_{t=1}^{4} \operatorname{card}\left(F_{t}^{1} \sqcup F_{t}^{2}\right)-\sum_{t=1}^{4} \operatorname{card}\left(F_{t}^{1} \cap F_{t}^{2}\right)= \\
(3+1+2+3)-(1+1+1+1)=5 .
\end{gathered}
$$

Having in mind that, in this case, the distance between two qualitative descriptions ranges from 0 to 16 , this value indicates that both qualitative descriptions are quite close.

\section{Modeling Group Preferences And Distances BETWEEN GROUPS}

The proposed model focusses the situation where the group of decision makers can be split into subgroups which are composed by members with similar profiles. In other words, there is a previous segmentation of the group of experts that is considered relevant for the stated decisionmaking problem. For instance, we can consider decisionmakers with different professional profiles, e.g. nurses, doctors or familiars in a health-care decision-making problem, or managers from different departments of a firm in a management decision-making problem.

The lattice structure of $\mathbb{S}_{n}$-fuzzy sets and the distance in Subsection II-C will allow us to deal with the subgroups' evaluations of features or alternatives in a group decisionmaking process. To this end, we consider the connected union in $\mathbb{S}_{n}$-fuzzy sets to model subgroups' evaluations.

Let $\Lambda$ be a set of features and $G$ a group of decision makers. Let $G=G_{1} \cup \cdots \cup G_{k}$, where each $G_{i}$ is a group of decision makers with a similar profile. Let $Q_{1}^{i}, \ldots, Q_{r_{i}}^{i}$ be the qualitative descriptions of $\Lambda$ provided by the decision makers in $G_{i}$.

Definition 9: For each $\lambda \in \Lambda$, the qualitative description of $\lambda$ corresponding to each $G_{i}$ is:

$$
Q_{G_{i}}(\lambda)=Q_{1}^{i}(\lambda) \sqcup \ldots \sqcup Q_{r_{i}}^{i}(\lambda)
$$

From this definition, the qualitative description of $\Lambda$ corresponding to the subgroup $G_{i}$ of decision makers, $i=i, \ldots, k$ is:

$$
\begin{aligned}
Q_{G_{i}}: & \Lambda \longrightarrow \mathbb{S}_{n} \\
& \lambda \mapsto Q_{G_{i}}(\lambda)=Q_{1}^{i}(\lambda) \sqcup \ldots \sqcup Q_{r_{i}}^{i}(\lambda)
\end{aligned}
$$

And these functions model the subgroups' linguistic preferences by means of $\mathbb{S}_{n}$-fuzzy sets. From these functions, the total group's linguistic preferences can be obtained as the qualitative description of $\Lambda$ corresponding to the total group $G$ of decision makers:

$$
\begin{aligned}
Q_{G}: & \Lambda \longrightarrow \mathbb{S}_{n} \\
\lambda & \mapsto Q_{G}(\lambda)=Q_{G_{1}}(\lambda) \sqcup \ldots \sqcup Q_{G_{k}}(\lambda) .
\end{aligned}
$$

The distance in $\mathbb{S}_{n}^{\Lambda}$ between qualitative descriptions, as defined in Subsection II-C, can be computed either for each pair of subgroups $G_{i}, G_{j}$ or for each subgroup $G_{i}$ and the total group $G$. These distances allow the analysis of the topology of the group of decision makers.

On the one hand, similarities and differences among subgroups' preferences are evidenced. To this end a matrix of distances between pairs $G_{i}, G_{j}$ can be computed.

On the other hand, similarities and differences between each subgroup and the total group of decision makers can also be revealed.

This knowledge is crucial in decision-making consensual processes that require several rounds of assessments to converge to a final solution. The topological analysis of the group of decision makers, allows to focus in the dissident subgroups. As a result, this analysis can significantly reduce the necessary number of rounds and the moderator's task.

\section{An Illustrative Example in the Retail Sector}

This section focuses on the empirical study that was conducted on a working session in which 70 senior managers from a major chain store organization participated. The objective of the study was to identify the most relevant features with regards to the performance of the firm. President Chain Corporation is a multinational retailing company operating in the regular chain convenience stores sector based in Taiwan. Managers were divided into four main subgroups depending on broad functional area: marketing (15); operations and store operations (17); accounting, finance and audit (24); R\&D and information systems (14). Previous to the working session, a state-of-the-art study and a set of in-depth qualitative interviews were conducted to identify 170 performancerelated variables in their sector. From this list, 44 features or variables related to resources used in retailing were selected as the main performance variables (see Table II).

\section{A. Data description}

An one-dimensional absolute order-of-magnitude model with 4 basic labels corresponding to the 4 ordered responses of the Likert scale used by the managers: (1) $\left\{a_{1}\right\}=$ extremely good; (2) $\left\{a_{2}\right\}=$ very good; (3) $\left\{a_{3}\right\}=$ moderately good; (4) $\left\{a_{4}\right\}=$ not very good. As a result we consider $\Lambda$ as the set of the 44 selected features. Data considered for this 


\begin{tabular}{|l|l|}
\hline Resource area & Feature \\
\hline Physical & $\lambda_{1}:$ Number of customer visits \\
resource & $\lambda_{2}:$ Store location \\
\hline Legal & $\lambda_{3}:$ Sales of private brand products \\
resource & $\lambda_{4}:$ Social responsibility \\
\hline Human & $\lambda_{5}:$ Employee turnover rate \\
resource & $\lambda_{6}:$ Staff training \\
\hline & $\lambda_{7}:$ Franchise system \\
& $\lambda_{8}:$ Store opening strategy \\
& $\lambda_{9}:$ Sales per store \\
& $\lambda_{10}:$ Spending-per-visit rate \\
& $\lambda_{11}:$ Internal procedures \\
& $\lambda_{12}:$ Achievement of year-end goals \\
& $\lambda_{13}:$ Investments in technology development \\
& $\lambda_{14}:$ Quality of data collection and process sys. \\
& $\lambda_{15}:$ Empowerment of staff \\
& $\lambda_{16}:$ Response to staff issues \\
& $\lambda_{17}:$ Inventory loss control \\
& $\lambda_{18}:$ Inventory service level \\
& $\lambda_{19}:$ Market positioning \\
& $\lambda_{20}:$ Store renovation/redecoration \\
& $\lambda_{21}:$ Expense control ability \\
& $\lambda_{22}:$ Percentage of part-time staff \\
resources & $\lambda_{23}:$ Shelf-life of new products \\
& $\lambda_{24}:$ Speed of new products development \\
& $\lambda_{25}:$ Past credit history \\
& $\lambda_{26}:$ Financial support from stockholders \\
& $\lambda_{27}:$ Internet channel development \\
& $\lambda_{28}:$ Maintaining target customers in market \\
& diversification \\
\hline External & $\lambda_{29}:$ Following fashion trends \\
factors & $\lambda_{30}:$ Facing seasonal demands \\
& $\lambda_{31}:$ Openness to criticism \\
& $\lambda_{32}:$ Willingness to innovate \\
\hline Relational & $\lambda_{33}:$ Customer complaints management \\
& $\lambda_{34}:$ Cost sharing with suppliers on promotions \\
& $\lambda_{35}:$ Joint venture opportunity with competitors \\
\hline resources & $\lambda_{36}:$ Changes in customer preferences \\
& $\lambda_{37}:$ Changes in supplier contract content \\
& $\lambda_{38}:$ Innovation and imitation from competitors \\
& $\lambda_{39}:$ Change in government laws \\
& $\lambda_{40}:$ Stability of government \\
& $\lambda_{41}:$ Innovation of new technology equipment \\
& $\lambda_{42}:$ New management system software devel. \\
& $\lambda_{43}:$ Change of population structure \\
& $\lambda_{44}:$ Change of lifestyle \\
\hline & \\
\hline & \\
&
\end{tabular}

study are the qualitative descriptions of $\Lambda$ provided by the 70 managers, considering the group of managers split into the four above-mentioned main subgroups.

The qualitative descriptions of the set $\Lambda$ given by managers are aggregated to obtain the qualitative descriptions corresponding to the different subgroups. For each group $G_{i}, i=$ $1, \ldots, 4$, the qualitative description $Q_{G_{i}}$ can be represented by means of a 44-dimensional vector of qualitative labels. Component $j$ of the vector $Q_{G_{i}}$, for each $j=1, \ldots, 44$, is the connected union of the responses of the managers with respect to the feature importance of the feature $\lambda_{j}$.

Let us consider, for instance, $G_{1}=$ "Department of Marketing", in this case $Q_{G_{1}}$ is represented by 44-dimensional vector of qualitative labels, containing four different labels:

$Q_{G_{1}}(\Lambda)=\left\{\left[a_{2}, a_{3}\right],\left[a_{2}, a_{4}\right],\left[a_{1}, a_{3}\right],\left[a_{1}, a_{4}\right]\right\}$ and the partition of $\Lambda$ associated to $Q_{G_{1}}$, i.e., the subsets of features described by these 4 qualitative labels are respectively:

$P_{1}=\left\{\left\{\lambda_{1}, \lambda_{2}, \lambda_{9}, \lambda_{10}, \lambda_{11}, \lambda_{12}, \lambda_{17}, \lambda_{18}, \lambda_{19}, \lambda_{20}, \lambda_{21}\right.\right.$, $\left.\lambda_{22}, \lambda_{23}, \lambda_{33}, \lambda_{44},\right\}\left\{\lambda_{3}, \lambda_{5}, \lambda_{6}, \lambda_{7}, \lambda_{13}, \lambda_{14}, \lambda_{15}, \lambda_{16}, \lambda_{27}\right.$, $\left.\lambda_{28}, \lambda_{31}, \lambda_{34}, \lambda_{35}, \lambda_{37}, \lambda_{39}, \lambda_{40}, \lambda_{41}, \lambda_{42}, \lambda_{43}\right\},\left\{\lambda_{4}, \lambda_{8}, \lambda_{24}\right.$,

\begin{tabular}{|l|l|l|l|l|l|}
\hline$D$ & $G_{1}$ & $G_{2}$ & $G_{3}$ & $G_{4}$ & $G$ \\
\hline$G_{1}$ & 0 & 0.87 & 0.57 & 0.67 & 0.63 \\
\hline$G_{2}$ & 0.87 & 0 & 0.9 & 1 & 0.7 \\
\hline$G_{3}$ & 0.57 & 0.9 & 0 & 0.83 & 0.57 \\
\hline$G_{4}$ & 0.67 & 1 & 0.83 & 0 & 0.9 \\
\hline
\end{tabular}

$\left.\left.\lambda_{25}, \lambda_{26}, \lambda_{29}, \lambda_{30}, \lambda_{32}, \lambda_{36}\right\},\left\{\lambda_{38}\right\}\right\}$.

Note that, when for a specific feature, managers in $G_{i}$ extremely disagree, the connected union of the managers' opinions in $G_{i}$ is the qualitative label $?=\left[a_{1}, a_{4}\right]$. In this case, only the feature $\lambda_{38}$ is described by ?. In addition, let us remark that few outlier managers in the group have been removed for some features (there was one outlier for features $\lambda_{4}, \lambda_{11}, \lambda_{18}, \lambda_{19}, \lambda_{20}, \lambda_{21}, \lambda_{39}, \lambda_{41}, \lambda_{42}, \lambda_{44}$ and two outliers for $\lambda_{29}$ ). Outliers have been determined using the median absolute deviation method [10], which in most cases corresponds to take the central $85 \%$ of data. Alternative approaches could be considered by taking the central 50\% o $75 \%$ of data, which would result in tighter intervals of data.

\section{B. Experimental results}

Considering the partitions of $\Lambda$ associated to $Q_{G_{i}}, i=$ $1, \ldots, 4$, corresponding to each one of the four departments, the partition $P=\left\{B_{1}, \ldots, B_{34}\right\}$ of $\Lambda$ such that all functions $Q_{G_{1}}, \ldots, Q_{G_{4}}$ are constant on each part $B_{i} \in \mathcal{P}, i=$ $1 \ldots, 34$, as constructed in [14] is:

$P=\left\{B_{1}=\left\{\lambda_{1}, \lambda_{2}, \lambda_{9}, \lambda_{12}, \lambda_{20}, \lambda_{23}\right\}, B_{2}=\right.$ $\left\{\lambda_{3},\right\}, B_{3}=\left\{\lambda_{4}\right\}, B_{4}=\left\{\lambda_{5}\right\}, B_{5}=\left\{\lambda_{6}, \lambda_{37}, \lambda_{43}\right\}, B_{6}=$ $\left\{\lambda_{7}\right\}, B_{7}=\left\{\lambda_{8}\right\}, B_{8}=\left\{\lambda_{10}\right\}, B_{9}=\left\{\lambda_{11}\right\}, B_{10}=$ $\left\{\lambda_{13}\right\}, B_{11}=\left\{\lambda_{14}\right\}, B_{12}=\left\{\lambda_{15}\right\}, B_{13}=$ $\left\{\lambda_{16}\right\}, B_{14}=\left\{\lambda_{17}, \lambda_{19}\right\}, B_{15}=\left\{\lambda_{18}\right\}, B_{16}=$ $\left\{\lambda_{21}\right\}, B_{17}=\left\{\lambda_{22}\right\}, B_{18}=\left\{\lambda_{24}\right\}, B_{19}=\left\{\lambda_{25}\right\}, B_{20}=$ $\left\{\lambda_{26}, \lambda_{32}\right\}, B_{21}=\left\{\lambda_{27}\right\}, B_{22}=\left\{\lambda_{28}, \lambda_{34}\right\}, B_{23}=$ $\left\{\lambda_{29}\right\}, B_{24}=\left\{\lambda_{30}\right\}, B_{25}=\left\{\lambda_{31}\right\}, B_{26}=\left\{\lambda_{33}\right\}, B_{27}=$ $\left\{\lambda_{35}\right\}, B_{28}=\left\{\lambda_{36}\right\}, B_{29}=\left\{\lambda_{38}\right\}, B_{30}=\left\{\lambda_{39}\right\}, B_{31}=$ $\left.\left\{\lambda_{40}\right\}, B_{32}=\left\{\lambda_{41}\right\}, B_{33}=\left\{\lambda_{42}\right\}, B_{34}=\left\{\lambda_{44}\right\}\right\}$.

And, considering $F_{i}^{j}=Q_{G_{i}}\left(B_{j}\right)$, the distances among departments are computed using $D_{Q}\left(Q_{G_{i}}, Q_{G_{j}}\right)=$ $\sum_{t=1}^{34} \operatorname{card}\left(F_{t}^{i} \sqcup F_{t}^{j}\right)-\sum_{t=1}^{34} \operatorname{card}\left(F_{t}^{i} \cap F_{t}^{j}\right)$. Their normalized values are summarized in Table III.

Results in Table III show that $G_{3}$, i.e. the department of accounting, finance and audit, is the most representative department of the total group of managers, being the one closer to $G$. Departments $G_{1}$, marketing, and $G_{3}$, accounting, finance and audit, are those that have expressed more similar opinions with respect to the 44 features related to the firm's performance. Finally, $G_{2}$, operations and store operations, and $G_{4}, \mathrm{R} \& \mathrm{D}$ and information systems, are the department with more different opinions compared to the rest.

\section{Conclusions}

This paper puts forward a method, based on a distance defined among groups of experts, for analyzing the topology 
of the group. Experts' evaluations are expressed using a set of linguistic labels describing order-of-magnitude. The method enables the handling of imprecise information given by evaluators. The approach has three main advantages. First, it takes into account the different degrees of strictness of the evaluators' opinions. Second, it removes the need to calculate an average value of ordinal data. Third, the method accommodates "unknown values" by using the label "?" defined in the absolute order-of-magnitude qualitative model.

From a well-ordered set $\mathcal{S}$ of basic labels, the extended set of qualitative labels $\mathbb{S}_{n}$ over $\mathcal{S}$ has been considered. The qualitative descriptions of a set $\Lambda$ are defined as $\mathbb{S}_{n}$-fuzzy sets. When there is a previous segmentation of the group of decision makers that is considered relevant for the stated decision-making problem, a $\mathbb{S}_{n}$-fuzzy set is defined for each subgroup of decision makers. A distance between $\mathbb{S}_{n}$-fuzzy sets allows us to analyze similarities and differences among subgroups and between each subgroup and the total group of decision makers.

A real-case application in the retail sector has been used to capture the differences between a firm's departments when assessing variables related to the performance of the firm. The real-case application gives us an example of how the model presented could benefit managerial decision-making processes.

Three main lines of future research are currently under consideration. First, using the concepts presented in this paper, to develop a web-based software tool capable of gathering and summarizing opinions and working simultaneously with different levels of precision for group decisionmaking processes. Second, the definition of a feedback process based on recommendations will be studied. These recommendations will be generated from rules induced by the distances between each subgroup and the total group. Finally, regarding the real case study, from the presented analysis that separately considers the functional area of managers, a study to improve consensus reaching will be addressed.

\section{REFERENCES}

[1] Agell, N. et al., 2012. Ranking multi-attribute alternatives on the basis of linguistic labels in group decisions. Information Sciences 209, 4960 .
[2] Deschrijver, G., Kerre, E.E. 2003. On the relationship between some extensions of fuzzy set theory, Fuzzy Sets and Systems 133, 227-235.

[3] Espinilla,M., Liu, J., L. Martínez, L. 2011. An extended hierarchical linguistic model for decision-making problems. Computational Intelligence 27 (3), 489-512.

[4] Forbus, K.D. 1984. Qualitative process theory, Artificial Intelligence $24(1-3), 85-168$.

[5] Goguen, J.A. 1967. L-fuzzy Sets, Journal of Mathematical Analysis and Applications 18, 145-174.

[6] Herrera, F., Herrera-Viedma, E., Martínez, L. 2008. A Fuzzy Linguistic Methodology to Deal with Unbalanced Linguistic Term Sets, IEEE Transactions on Fuzzy Systems 16 (2), 354-370.

[7] Herrera-Viedma, E., Herrera, F., Chiclana, F. 2002. A consensus model for multiperson decision making with different preference structures. IEEE Transactions on Systems, Man and Cybernetics, Part A: Systems and Humans 32, 394-402.

[8] Herrera-Viedma, E., Cabrerizo, F., Kacprzyk, J., Pedrycz, W. 2014. A review of soft consensus models in a fuzzy environment. Information Fusion 17, 4-13.

[9] Huang, H.-L., Shi,F.-G. 2008. L-fuzzy numbers and their properties, Information Sciences 178, 1141-1151.

[10] Leys, C., Ley, C., Klein, O., Bernard, P., Licata, L. 2013. Detecting outliers: Do not use standard deviation around the mean, use absolute deviation around the median, Journal of Experimental Social Psychology 49(4), 764-766.

[11] Meng, L., Wang, X., Kerre, E. 2010. An improved representation theorem of L-fuzzy sets, Fuzzy Sets and Systems 161, 3141-3147.

[12] Negoita, C.V., Ralescu, D.A. 1975 Applications of Fuzzy Sets to Systems Analysis. Stuttgart and Halsted Press, New York.

[13] Parreiras, R.O., Ekel, P.Ya., Martini, J.S.C., Palhares, R.M. 2010. A flexible consensus scheme for multicriteria group decision making under linguistic assessments, Information Sciences 180 (7), 1075-1089.

[14] Prats, F., Roselló, L., Sánchez, M., Agell, N. 2014. Using L-fuzzy sets to introduce information theory into qualitative reasoning. Fuzzy Sets and Systems 236 (1), 73-90.

[15] Rodríguez, R.M., Martínez, L., Herrera, F. 2012. Hesitant fuzzy linguistic terms sets for decision making, IEEE Transactions on Fuzzy Systems 20 (1), 109-119.

[16] Roselló, L., Prats, F., Agell, N., Sánchez, M. 2010. Measuring consensus in group decisions by means of qualitative reasoning. International Journal of Approximate Reasoning 51, 441-452.

[17] Seselja, B., Tepavcevic, A. 2003. Representing ordered structures by fuzzy sets: an overview, Fuzzy Sets and Systems 136, 21-39.

[18] Shi, F.-G. 2009. L-fuzzy interiorsand $L$-fuzzy closures, Fuzzy Sets and Systems 160, 1218-1232.

[19] Tang, Y., Zheng, J. 2006. Linguistic modelling based on semantic similarity relation among linguistic labels, Fuzzy Sets and Systems 157 (12), 1662-1673.

[20] L. Travé-Massuyès, P. Dague, L. Ironi. 2003. Mathematical foundations of qualitative reasoning, AI Magazine 24 (4), 91-106.

[21] L. Travé-Massuyès, F. Prats, M. Sánchez, N. Agell. 2005. Relative and absolute order-of-magnitude models unified, Annals of Mathematics and Artificial Intelligence 45 (1), 323-341.

[22] Wang, G.-j., He, Y.-Y. 2000. Intuitionistic fuzzy sets and $L$-fuzzy sets, Fuzzy Sets and Systems 110, 271-274. 